\title{
Ocular motility as an indicator of verbal and visuospatial processing
}

\author{
MERRILL HISCOCK and KATHLEEN J. BERGSTROM \\ University of Saskatchewan, Saskatoon, Saskatchewan S7N OWO, Canada
}

\begin{abstract}
Three experiments were conducted to study ocular motility as a function of cognitive task. Horizontal eye movements were recorded electrically as normal adults attempted to answer auditorily presented questions that were either verbal-conceptual or visuospatial in nature. In each of the three experiments, verbal-conceptual questions elicited significantly more eye movements than did visuospatial questions. Direction of initial movement was a less reliable indicator of question category. Experiment 1 showed that the difference in eye movement rate associated with question category persisted throughout the period of deliberation; in Experiment 2, consistent differences in ocular motility were obtained with diverse verbal-conceptual and visuospatial questions; Experiment 3 showed that the effect is found even when an oral answer is not required. These findings cannot be attributed to differences in task difficulty or response factors. A low eye movement rate may facilitate visual thinking by reducing interference from the environment, or it may reflect bilateral activation of the cerebral hemispheres.
\end{abstract}

Eye movements of fixation, or saccades, have been studied primarily in the context of visual information acquisition (Rayner, 1978; Robinson, 1968). Nevertheless, it is well documented that saccadic eye movements occur in humans when there is little or no visual information to be acquired from the environment (Day, 1964; Kinsbourne, 1972; Moore, 1903; Teitelbaum, 1954). These stimulus-independent eye movements usually have been explained as motor concomitants of mental processes or of changes in physiological state, but attempts to identify endogenous correlates of these movements have yielded inconsistent and even contradictory results.

It has been reported that eye movement frequency varies as a function of specific cognitive demands (Brown, 1968; Lorens \& Darrow, 1962; Marks, 1972), but other studies implicate nonspecific factors such as attention or effort as determinants of ocular motility (Amadeo \& Gomez, 1966; Amadeo \& Shagass, 1963; Brady \& Rosner, 1966; Weitzenhoffer \& Brockmeier, 1970). Even among studies that are in agreement as to the nature of the factors influencing ocular motility, the actual relationship between these factors and eye movement frequency is reversed in some studies relative to others. For instance, contrary to most studies, Weitzenhoffer and Brockmeier (1970) reported that attention-demanding tasks caused a reduction in eye movement rate (EMR) relative to a baseline rate. Sim-

This work was supported by grants from the University of Saskatchewan and the Medical Research Council of Canada. The authors are grateful to C. A. Burnham, E. A. Clark, D. B. Cohen, J. M. Swanson, and T. B. Wishart for their advice and assistance. Requests for reprints should be sent to Merrill Hiscock, Division of Psychology, University Hospital, Saskatoon, Saskatchewan S7N 0X0, Canada. ilarly, the use of visual imagery has been associated with both increased (Brown, 1968; Lorens \& Darrow, 1962) and decreased (Marks, 1972) rates of ocular activity.

Ocular motility during task performance usually has been compared with ocular motility during a baseline period in which no task is presented. Since it is impossible to ascertain what cognitive activity actually is taking place during the baseline period, motility differences between the baseline condition and any other condition do not lend themselves to unambiguous interpretation. Moreover, the cognitive tasks frequently elicited no observable behavior that could serve as verification that the intended cognitive processing occurred. In an attempt to avoid these methodological pitfalls, Weiner and Ehrlichman (1976) compared EMRs as subjects answered questions that were either verbal-conceptual or visuospatial in nature. They found that verbal-conceptual questions elicited significantly greater ocular motility than did visuospatial questions. This finding raises the possibility that a reliable relationship between cognitive process and ocular motility may be found if an appropriate methodology is used.

The present report describes a series of three experiments designed to test Weiner and Ehrlichman's (1976) claim that verbal and visuospatial processes can be differentiated on the basis of ocular motility. In each of these studies, electrooculography (EOG) was used to provide a detailed and objective record of ocular motility during the period following each question. Diverse verbal-conceptual and visuospatial questions were used in an attempt to determine the generality of type-ofquestion effects; performance levels and response latencies were analyzed; and the requirement of a vocal response was manipulated in an attempt to eliminate a possible artifact. In addition, the direction of the first 
lateral eye shift was scored in an attempt to confirm sporadic findings of a relation between type of question and direction of eye movement (Ehrlichman \& Weinberger, 1978; Ehrlichman, Weiner, \& Baker, 1974; Kinsbourne, 1972, 1974; Kocel, Galin, Ornstein, \& Merrin, 1972).

\section{EXPERIMENT 1}

The primary objectives of the first experiment were (1) to confirm Weiner and Ehrlichman's (1976) finding that verbal-conceptual questions elicit more eye movements than do visuospatial questions and (2) to determine whether this task-related difference in ocular motility occurs only at one point in time or whether it persists throughout the period during which the question is being deliberated. For comparison purposes, eye movements also were recorded following the presentation of instructions to generate specified auditory and visual images. If visuospatial processing is associated with relative quiescence of the eyes, this association would constitute strong evidence against the imagery-scanning hypothesis of eye movements (Hebb, 1968; Totten, 1935).

The influence of cognitive task upon direction of the initial eye movement also was investigated. Kinsbourne $(1972,1974)$ has proposed that gaze shifts to the right or to the left reflect a tendency to turn in a direction opposite to the more highly activated cerebral hemisphere. Insofar as the left hemisphere is specialized for verbal processing and the right hemisphere is specialized for visuospatial processing, it follows that verbal questions should evoke predominantly rightward eye movements and that visuospatial questions should evoke predominantly leftward movements. Unfortunately, investigations of the lateral eye movement phenomenon have yielded equivocal results (see Ehrlichman \& Weinberger, 1978). Experiment 1 provides an opportunity to observe an association between the nature of the task and the direction of the initial eye movement.

\footnotetext{
Method

Subjects. Subjects were 27 university undergraduates who volunteered to participate in the study. Fourteen subjects were right-handers (eight females, six males) and 13 were left-handers (six females, seven males).

Materials. Five verbal and five visuospatial questions were used to elicit eye movements. The verbal questions consisted of three proverbs and two similarities items from the verbal scale of the Wechsler Adult Intelligence Scale (WAIS); the visuospatial items were questions such as, "You are standing on your head, facing east; you swivel $90 \mathrm{deg}$ to your right; which direction are you now facing?" In addition, subjects were administered five visual imagery tasks (e.g., "Imagine the face of a friend") and five auditory imagery tasks (e.g., "Imagine the sound of escaping steam").

Apparatus. Lateral eye movements were recorded on a Beckman Type RS dynograph with direct nystagmus coupler (Type 9859). Recording was bipolar: Beckman miniature surface electrodes were placed at the outer canthi and a reference electrode was placed $2 \mathrm{~cm}$ above the nasion. AC amplification was used [ time constant $(\mathrm{TC})=3 \mathrm{sec}]$.
}

Procedure. Testing took place in a normally illuminated room. To ensure spontaneity of eye movements, the subject was told that frontal electroencephalography (EEG), rather than EOG, was being recorded. The subject sat facing a homogeneous white field at a distance of approximately $30 \mathrm{~cm}$. A head restraint prevented any noticeable head movements. The experimenter and recording apparatus were situated $1.5 \mathrm{~m}$ directly behind the subject.

After the experimenter attached the electrodes, the dynograph was calibrated by having the subject fixate three black markings on a cord suspended in front of his or her eyes. Gain was adjusted so that a lateral eye movement of 10 deg produced a pen deflection of $1 \mathrm{~cm}$.

Lateral eye movements were then recorded as the subject attempted to answer the 10 auditorily presented questions. The experimenter read the questions at 20 -sec intervals in the same randomized order for all subjects. Answers were given orally. The experimenter marked the EOG record to show the beginning and end of each question, as well as the onset of the subject's response. Following presentation of the 10 questions, the subject was asked to form the $\mathbf{1 0}$ different auditory and visual images. No overt response to the imagery items was required.

Scoring. EOG records were scored blindly with respect to question category. Saccadic deflections were scored as eye movements only if they met or exceeded an amplitude criterion of $.5 \mathrm{~cm}$, which represented a 5-deg horizontal component. Scoring for the verbal and visuospatial questions began $1 \mathrm{sec}$ prior to the end of the question and continued until $1 \mathrm{sec}$ prior to the vocal response or until $13 \mathrm{sec}$ had elapsed without a response. Eye movements associated with imagery items were scored for a 13-sec interval beginning $1 \mathrm{sec}$ prior to the end of the instruction. Additionally, for each verbal and visuospatial question, the first deflection after the end of the question that met or exceeded the amplitude criterion was scored as either a right or left lateral eye movement.

For the purpose of estimating interrater agreement, a second scorer independently scored each subject's eye movements for one randomly selected verbal question and one randomly selected visuospatial question.

\section{Results}

The primary dependent variable was mean EMR, expressed in eye movements per second $(\mathrm{EM} / \mathrm{sec})$. A preliminary analysis of variance showed that neither subjects' sex nor handedness had a significant effect on EMR ( $p>.15$ ). Moreover, neither of these individual difference variables interacted with question category $(p>.15)$. Consequently, for subsequent analyses, subjects were pooled irrespective of these classifications.

Independently scored rates for the randomly selected verbal and visuospatial questions were compared to assess interrater agreement. Correlations between results from the two scorers were .98 for the verbal question and .99 for the visuospatial question.

Mean EMR for each task and each phase of recording is shown in Table 1. A one-way analysis of variance performed on the composite scores shown in the last column of Table 1 yielded a significant effect for question category $[F(3,78)=35.69, p<.0001]$. By NewmanKeuls test, verbal questions elicited more frequent eye movements than did visuospatial questions $(p<.01)$, and both verbal and visuospatial questions elicited more oculomotor activity than did either the visual or auditory imagery instructions $(p<.01)$. Rates associated 
Table 1

Eye Movement Rate (in Eye Movements per Second) Associated With Each of Four Tasks

\begin{tabular}{|c|c|c|c|c|c|c|c|c|c|c|c|c|}
\hline \multirow[b]{3}{*}{ Task Category } & \multicolumn{10}{|c|}{ Time From End of Question or Instruction (in Seconds) } & & \\
\hline & \multicolumn{2}{|c|}{-1 to 0} & \multicolumn{2}{|c|}{0 to 1} & \multicolumn{2}{|c|}{1 to 2} & \multicolumn{2}{|c|}{2 to 3} & \multicolumn{2}{|c|}{$>3$} & \multicolumn{2}{|c|}{ Composite* } \\
\hline & Mean & $\mathrm{SD}$ & Mean & SD & Mean & SD & Mean & $\mathrm{SD}$ & Mean & SD & Mean & SD \\
\hline Verbal Questions & .748 & .672 & 1.052 & .720 & 1.120 & .710 & 1.069 & .597 & 1.033 & .526 & 1.045 & .539 \\
\hline Visuospatial Questions & .578 & .572 & .711 & .603 & .740 & .663 & .494 & .522 & .607 & .498 & .679 & .463 \\
\hline Auditory Imagery & .400 & .426 & .370 & .446 & .274 & .364 & .207 & .263 & .293 & .304 & .292 & .275 \\
\hline Visual Imagery & .541 & .663 & .415 & .493 & .259 & .327 & .237 & .360 & .282 & .310 & .307 & .299 \\
\hline
\end{tabular}

*Based on total number of scorable eye movements after each question or instruction, divided by number of seconds scored, beginning with $t=0$.

with each of the two kinds of imagery items did not differ significantly.

Subsequent analyses of variance were performed to assess changes in EMR across different phases of the tasks. Data for the questions and for the imagery items were analyzed separately. The analyses were 2 by 4 repeated-measures analyses of variance with task category (verbal vs. visuospatial questions or auditory vs. visual imagery items) and phase as independent variables. Since verbal and visuospatial questions usually were answered between 3 and $12 \mathrm{sec}$ after the end of the question (mean $=5.46 \mathrm{sec}$ ), only the time from $1 \mathrm{sec}$ prior to the end of the question until $3 \mathrm{sec}$ after the end of the question was considered. The analysis for verbal and visuospatial questions yielded a significant main effect for question category $[F(1,26)=17.11, p<.0005]$ and a significant main effect for phase $[F(3,78)=3.59$, $p<.025]$. The phase effect was attributable primarily to the quadratic trend component $[\mathrm{F}(1,26)=7.44$, $\mathrm{p}=.01]$. Ocular motility was greater in the first $2 \mathrm{sec}$ following the end of the question than in the preceding or subsequent $1-\mathrm{sec}$ period. The Phase by Question Category interaction was nonsignificant $[F(3,78)=1.67$, $p>.15]$, which suggests that the difference between verbal and visuospatial questions in ocular motility did not change appreciably throughout the period of deliberation.

An identical analysis of variance for the imagery tasks yielded only a significant main effect for phase $[F(3,78)$ $=5.75, \mathrm{p}<.001]$ that can be attributed to the linear trend component $[F(1,26)=10.54, p<.005]$. EMR decreased linearly during the $4-\mathrm{sec}$ period that began $1 \mathrm{sec}$ prior to the end of the instruction.

The mean percentage of correct answers for verbal and for visuospatial questions did not differ significantly (mean $=31.1 \%$ for verbal questions, mean $=33.3 \%$ for visuospatial questions; $\mathrm{F}<1$ ). However, the average response latency was significantly greater for verbal questions (mean $=6.98 \mathrm{sec}$ ) than for visuospatial questions (mean $=3.95 \mathrm{sec}$ ) $[\mathrm{F}(1,26)=48.32, \mathrm{p}<.0001]$.

Verbal questions yielded an average of 4.74 trials/sub. ject with at least one scorable eye movement. Initial movements were rightward in $50.78 \%$ of those trials. Visuospatial questions yielded an average of 3.78 trials/ subject with at least one eye movement, and $30.39 \%$ of these movements were rightward. The difference in percentage of rightward movements was statistically significant $[F(1,22)=8.08, p<.01]$. Neither subjects' sex nor handedness had a significant effect on the proportion of rightward eye movements $(F<1)$, and neither of those factors interacted with question category $(F<1)$.

\section{Discussion}

Consistent with Weiner and Ehrlichman's (1976) finding, verbal-conceptual questions were associated with a dramatically higher saccadic rate than were visuospatial questions. The peak in ocular motility occurred during the first $2 \mathrm{sec}$ after the end of the questions, but the verbal vs. spatial differences in EMR appeared to be fairly constant over the entire 4-sec interval that was examined. Difficulty level cannot account for the type-of-question effect if difficulty is defined in terms of the proportion of correct responses. However, the average difference between latency to respond to verbal questions and latency to respond to visuospatial questions leaves open the possibility that verbal items are more difficult than visuospatial ones. The imagery tasks yielded EMRs substantially lower than that for either verbal or visuospatial questions, but there was no difference in EMR as a function of imagery modality. Because the imagery tasks did not require vocal response, the low EMR might be attributed to a low arousal level (Amadeo \& Shagass, 1963).

The results of the present study are in direct opposition to the imagery-scanning hypothesis. Rather than producing ocular motility, tasks designed to elicit visual imagery were accompanied by relative quiescence of the eyes. The low EMR following instructions for auditory imagery was unexpected. It is possible that the auditory items (e.g., "Imagine the sound of escaping steam") elicited visual as well as auditory images. The primary shortcoming of such imagery tasks is the lack of independent verification that the intended cognitive processes actually did occur.

In agreement with past findings (Kinsbourne, 1972; Kocel et al., 1972), verbal questions produced more rightward eye movements than did visuospatial questions. 


\section{EXPERIMENT 2}

A second experiment was designed to replicate and extend Experiment 1. The primary objective was to ascertain the generality of the verbal vs.visuospatial difference in EMR. Task difficulty and response latency again were considered as possible artifacts. In addition, the relation between type of question and direction of eye movement again was investigated.

\section{Method}

Subjects. Twenty-seven male undergraduate psychology students participated in the study. All subjects were righthanders.

Materials. The stimuli used to elicit eye movements were 20 verbal and 20 visuospatial questions. The verbal questions consisted of eight proverbs to be interpreted, four similarities items from the WAIS, four word definitions, and four miscellaneous verbal questions (Kocel et al., 1972). Examples of the miscellaneous questions are: "Name two conjunctions" and "Name a word with three syllables." Ten of the visuospatial questions required "static" visual memory (e.g., "How many rows of keys are there on a typewriter keyboard?"); the other 10 visuospatial questions required some further manipulation or elaboration (e.g., "If the rising sun is at your back, what direction will you be facing if you turn $90 \mathrm{deg}$ to your right?").

Questions were assigned randomly to blocks of 10 verbal and 10 visuospatial questions, with the constraint that questions in a particular category (e.g., proverbs) be divided equally between the two appropriate blocks (i.e., between the two verbal or the two visuospatial blocks). The blocks were presented in four different counterbalanced orders such that verbal and visuospatial blocks always were alternated. The interval between questions within a block was $20 \mathrm{sec}$; the interval between blocks was $60 \mathrm{sec}$.

Apparatus. An E\&M Model DMP-4A physiograph was used to record horizontal eye movements. A direct-coupled (d-c) preamplifier made it possible to obtain continuous eye position information. As in Experiment 1, bipolar recording was used, but in Experiment 2 the reference electrode was attached to the right earlobe instead of the forehead.

Procedure. The experimental setting and procedures were similar to those of the first experiment, with the following exceptions. In Experiment 2, the experimenter and recording apparatus were located in a room adjacent to the room in which the subject was seated. Questions were delivered via tape recorder, and oral responses were transmitted to the experimenter via intercom. To calibrate the recording apparatus, subjects were instructed to fixate small colored triangles on a chart placed $61 \mathrm{~cm}$ in front of their eyes. Gain was adjusted so that a 10-deg lateral eye movement yielded a $5-\mathrm{mm}$ pen deflection.

Scoring. As in Experiment 1, eye movements were scored by one of the authors, who remained blind with respect to question category. Pen deflections of $2.5 \mathrm{~mm}$ or more were counted if they occurred after the end of a question and at least $1 \mathrm{sec}$ prior to the subject's oral response. This criterion corresponded to an eye movement with a horizontal component of at least $5 \mathrm{deg}$. In the event of no oral response, eye movements were scored during the $12 \mathrm{sec}$ following the end of the question.

Interrater agreement estimates were based on independent, blind scoring by the two authors of two randomly chosen verbal questions and two randomly chosen visuospatial questions.

\section{Results}

Correlations between the independently derived EMR scores were .95 for each of the two verbal questions, and .99 and .98 , respectively, for the two visuospatial questions.
Table 2

Eye Movement Rate (in Eye Movements per Second) Associated With Each of Four Verbal-Conceptual Tasks and Each of Two Visuospatial Tasks

\begin{tabular}{lcc} 
& \multicolumn{2}{c}{ Rate } \\
\cline { 2 - 3 } \multicolumn{1}{c}{ Task } & Mean & SD \\
\hline Proverbs & .688 & .339 \\
WAIS Similarities & .793 & .423 \\
Word Definitions & .727 & .473 \\
Miscellaneous Verbal Questions & .687 & .330 \\
Visual Memory & .367 & .255 \\
Elaborated Visuospatial & .377 & .287 \\
\hline
\end{tabular}

The mean EMR for each of the six question categories is given in Table 2. A one-way analysis of variance showed that there was a significant difference in EMR among categories $[F(5,130)=18.11, p<.0001]$. The Newman-Keuls test indicated that the EMR associated with each of the four verbal categories was significantly greater than that associated with each of the two visuospatial categories, at the .01 level of probability. None of the rates associated with verbal questions differed from any of the others, and rates associated with the two visuospatial categories did not differ significantly.

Although there was a significant difference among question categories in the percentage of correct answers given $[F(5,130)=8.56, p<.0001]$, there was no average difference between verbal (mean $=59.2 \%$ ) and visuospatial questions (mean $=62.6 \%) \quad(\mathrm{F}<1)$. The significant category effect reflects the fact that proverbs were significantly more difficult (mean $=41.9 \%$ correct) than all of the other question types except WAIS similarities and that similarities were more difficult than definitions and miscellaneous verbal questions.

Response latency data showed a pattern similar to that of the correct response data. There was a significant main effect for question category $[F(5,130)=6.04$, $\mathrm{p}<.0001]$, but Newman-Keuls tests revealed that the only significant latency differences were those between proverbs (mean $=8.02 \mathrm{sec}$ ) and each of the other categories $($ mean $=6.59 \mathrm{sec})$.

Verbal questions, irrespective of category, yielded an average of 18.07 trials/subject with at least one scorable eye movement; $50.41 \%$ of the initial movements were rightward. Visuospatial questions yielded an average of 14.37 trials/subject with at least one eye movement; $51.03 \%$ of the initial movements were rightward. There was no significant difference between question categories in the percentage of rightward movements $(t<1)$.

\section{Discussion}

The verbal vs. visuospatial difference in EMR was found with all combinations of verbal and visuospatial questions. Therefore, the difference appears to be a general one that is not restricted to particular kinds of questions. Because the effect is found with heterogeneous questions, the difference in EMR is not likely to be an artifact of question length, syntax, or some other 
property of specific kinds of questions. Although some kinds of questions were more difficult on the average than others or required longer processing times, these differences cannot account for the EMR differences. EMR differences between verbal and visuospatial questions were observed even when performance and response latency were equivalent.

No difference in EMR was found between visuospatial questions presumed to require "static" memory and those presumed to require cognitive elaboration (rotation, juxtaposition, etc.). The failure of "kinetic" visual imagery tasks to produce substantial ocular motility constitutes further evidence against the imagery. scanning hypothesis (Hebb, 1968; Totten, 1935).

Despite the similarity in methodology to Experiment 1 and despite the large number of questions used in Experiment 2, verbal and visuospatial questions elicited an equivalent percentage of rightward eye movements in the second experiment. The difficulty in consistently demonstrating an association between cognitive task and direction of initial eye movement casts doubt on the utility of reflective lateral eye movements as indicators of hemispheric asymmetry (see Ehrlichman \& Weinberger, 1978). Possibly minor methodological alterations, such as blocking similar questions instead of randomizing them, are sufficient to preclude finding the predicted effect.

\section{EXPERIMENT 3}

A third experiment was conducted in order to (1) further explore the role of response factors and (2) confirm the previous finding that tasks requiring mental rotation or other elaboration produce no more eye movements than do "static" visualization tasks.

\section{Method}

Subjects. Sixteen university undergraduates (10 females, 6 males) served as subjects. All but two were right-handed.

Materials. There were 10 questions in each of three categories: verbal (word definitions, proverbs, WAIS similarities), "static" visual memory, and elaborated visuospatial.

Apparatus. Eye movements were recorded on a Grass Model III recorder with ac amplification (TC $=3 \mathrm{sec}$ ).

Procedure. The experimental setting and procedures were similar to those of the second experiment. The 10 questions within each of the three categories were divided into two matched sets. Subjects were asked to answer each question within one set but to respond to the other set of questions by saying "okay" when they had determined what the answer should be. Eight subjects heard the 15 questions requiring an oral answer first; the other eight subjects heard the other 15 questions first. Within each set of 15 questions, items were blocked according to category (i.e., verbal, static visual memory, elaborated visuospatial).

Scoring. Scoring criteria for eye movements were identical to those in Experiment 1, except that movements were scored only if they occurred from $1 \mathrm{sec}$ prior to the end of the question until $2 \mathrm{sec}$ after the end of the question. The first eye movement occurring in the 3-sec interval was scored as either right or left.
Table 3

Eye Movement Rate (in Eye Movements per Second) as a Function of Question Category and Response

\begin{tabular}{|c|c|c|c|c|}
\hline \multirow{3}{*}{$\begin{array}{l}\text { Question } \\
\text { Category }\end{array}$} & \multicolumn{4}{|c|}{ Response } \\
\hline & \multicolumn{2}{|c|}{ Oral Answer } & \multicolumn{2}{|c|}{ "Okay" } \\
\hline & Mean & SD & Mean & SD \\
\hline Verbal & .963 & .534 & .700 & .507 \\
\hline Visual Memory & .496 & .401 & .413 & .366 \\
\hline Elaborated Visuospatial & .571 & .407 & .400 & .349 \\
\hline
\end{tabular}

\section{Results}

Table 3 shows the mean EMR for each question category and response condition. The data were analyzed in a 3 by 2 repeated-measures analysis of variance with question category and response condition as the respective design factors. The analysis yielded a significant main effect for question category $[F(2,30)=20.74$, $p<.0001]$ that is attributable to the verbal vs. pooled visuospatial contrast $[F(1,15)=29.27, p<.0001]$. Verbal questions yielded a higher EMR than did visuospatial questions. The EMR associated with "static" visual memory questions and elaborated visuospatial questions did not differ significantly $(F<1)$. A significant main effect for response mode $[F(1,15)=13.02$, $p<.005]$ indicates that questions requiring an oral answer elicited greater motility than did questions not requiring an oral answer. The Question Category by Response Mode interaction was nonsignificant $(F<1)$.

For the 15 questions requiring answers, the percentage of questions answered correctly (mean $=51.7 \%$ ) did not differ significantly across question categories $(F<1)$.

When all questions were considered, there was a significant difference across categories in response latency $[F(2,30)=25.11, p<.0001]$. "Static" visual memory questions elicited faster responses (mean $=4.88 \mathrm{sec}$ ) than did either elaborated visuospatial questions (mean $=$ $6.20 \mathrm{sec}(\mathrm{p}<.01)$ or verbal questions (mean $=6.97 \mathrm{sec})$ $(p<.01)$. However, there was no significant difference between the latency to answer elaborated visuospatial questions and that to answer verbal questions. Latencies for oral answers (mean $=5.99 \mathrm{sec}$ ) did not differ significantly from latencies for responding "okay" when an answer had been reached (mean $=6.04 \mathrm{sec})(\mathrm{F}<1)$.

At least one eye movement occurred on an average of 8.00 of the 10 verbal trials; $54.69 \%$ of the initial movements were rightward. Of the 20 visuospatial trials, the mean number that yielded at least one eye movement was $11.13 ; 47.19 \%$ of the initial movements were to the right. The difference in percentage of rightward movements for the two kinds of question was nonsignificant $(\mathrm{t}<1)$.

\section{Discussion}

The verbal vs. visuospatial difference in EMR is independent of the requirement for a vocal answer. 
Although the EMR was higher for questions requiring a vocal answer than for similar questions requiring no answer, verbal questions produced greater motility than did visuospatial questions irrespective of the response factor. This result constitutes strong evidence that the question category effect is not an artifact of some unspecified difference in response characteristics between verbal and spatial questions. Nevertheless, the significant effect of response requirement on EMR is consistent with an arousal explanation for ocular motility (Amadeo $\&$ Shagass, 1963). The results suggest that the verbal vs. visuospatial nature of the task as well as arousal level may exert independent influences on eye movement frequency.

As in Experiment 2, there was no significant difference between EMRs associated with static and kinetic imagery questions. Thus, the findings again fail to support the imagery-scanning hypothesis.

\section{GENERAL DISCUSSION}

The results of all three experiments confirm Weiner and Ehrlichman's (1976) report that verbal-conceptual questions elicit a higher rate of saccadic activity than do visuospatial questions. Even though EOG was used rather than videotape to record eye movements, Weiner and Ehrlichman's basic findings were duplicated using a wide variety of visuospatial and verbal questions. The magnitude of the verbal vs. visuospatial question effect was consistently large; in some instances, verbalconceptual questions elicited more than twice as many eye movements per second as did visuospatial questions. In addition, the data show that verbal vs. visuospatial differences in EMR persist from the last second of the question until at least $1 \mathrm{sec}$ before an answer is given. These task-related differences in EMR are independent of response latency and question difficulty as inferred from proportion of correct responses. In fact, the differences are found even when no answer to the question is given.

Findings of the present study are inconsistent with the imagery-scanning hypothesis of eye movement (Hebb, 1968; Totten, 1935). It could be argued that the scanning of static images might not require gaze shifts as large as $5 \mathrm{deg}$, but that argument becomes less plausible as the imagery becomes more "kinetic." More prominent saccades should accompany the mental manipulation (rotation, juxtaposition, etc.) of images if the scanning hypothesis is correct. Thus, the failure to find any difference in ocular motility between the static and elaborated imagery conditions seems clearly incompatible with the imagery-scanning hypothesis.

Either of two alternative models can account for results of the present study more satisfactorily than the imagery-scanning model. One of these models, favored by Weiner and Ehrlichman (1976) in explaining their results, is based on the notion of selective interference between external and internal visual stimuli. The other model is the orientational model that previously has been used to explain asymmetries in lateral eye movements (Kinsbourne, 1972, 1974).

There is some evidence that visual input from the environment and centrally generated visual stimuli (i.e., images) compete for the processing resources of a limited-capacity visual "channel" (Atwood, 1971; Bower, 1970; Brooks, 1968). It follows that ocular quiescence may facilitate visual imagery insofar as it reduces interference from the external environment. In fact, it has been shown that subjects tend to suppress their oculomotor response to a compelling visual stimulus when they are engaged in certain cognitive activities that presumably involve mental imagery (Singer, Greenberg, \& Antrobus, 1971).

Although the selective interference, or channelcapacity, model can account for the major findings of the present study, there are two aspects of the results that appear anomalous with respect to the model. First, in Experiment 1, visual and auditory imagery instructions yielded equivalent levels of ocular motility. On the basis of the selective interference model, visual imagery should be associated with a lower EMR than should auditory imagery. However, as noted previously, it seems quite likely that instructions to form auditory images resulted in the formation of visual, as well as auditory, images. Second, the results of the first and third experiments imply that questions requiring the formulation of answers, irrespective of their content, elicit relatively high EMRs compared with tasks that either are easier or require less complete processing. This finding is more consistent with an attention- or effort-based model of EMR than with the selective interference model. On the other hand, there is no evidence in any of the three experiments that EMR is related to the difficulty of the task, as assessed on the basis of response latency and level of performance. In Experiment 3, for instance, static visual memory questions were answered significantly more quickly than were elaborated visuospatial questions, but there was virtually no difference between the two categories of question in EMR.

Kinsbourne's $(1972,1974)$ orientational model offers a neuropsychological explanation for the question category effect on EMR that was observed consistently in the present study. The model usually is invoked to explain asymmetries in the direction of reflective lateral eye movements, in which case it is assumed that verbal. conceptual questions activate primarily the left cerebral hemisphere and visuospatial questions activate primarily the right hemisphere. Under certain circumstances, which apparently are difficult to specify and to duplicate (see Ehrlichman \& Weinberger, 1978), lateral eye movement direction seems to conform to the pattern predicted on the basis of that assumption. However, there are at least two reasons why the laterality effect might be unreliable. First, when the subject is asked questions in a situation other than face-to-face confrontation, there is not one clear-cut gaze shift but, rather, a multiplicity of eye movements (Hiscock, 
1977). Consequently, it is difficult to identify the "significant" initial movement. Second, since visuospatial questions are presented verbally and answered verbally, they cannot be considered as pure examples of a visuospatial task. EMR, which does not rely entirely on one key movement, may reflect relative left- and right-hemispheric contributions to cognitive processing in the following manner. If verbal-conceptual questions are processed primarily by the left hemisphere, it seems likely that the activation level of the left hemisphere will fluctuate during solution of the problem. Increasing activation will cause rightward eye movements, but decreasing activation will cause the eyes to return to midline or to deflect to the left (see Kinsbourne, 1974). Although the initial movement should be a rightward movement, it is difficult to specify when that movement will occur. Subsequent movements may be either rightward or leftward. Because of the mixed verbal and visuospatial nature of the nominally visuospatial questions, their processing is likely to be divided more equally between the two cerebral hemispheres. If so, both hemispheres may be activated simultaneously, and it has been shown that simultaneous stimulation of the two hemispheres causes the eyes to remain on the midline (Crosby, 1953). This interpretation of Kinsbourne's model leads to the prediction that a high frequency of vertical eye movements will be associated with visuospatial questions. Although vertical movements were not measured in the present study, a previous experiment did show that spatial questions elicit a large number of vertical gaze shifts (Kinsbourne, 1972).

Either the selective interference model or the orientational model as interpreted above can account for most of the data from the present study, and there is little reason to favor one or the other in the absence of further evidence. In addition, some aspects of the present findings suggest that arousal or effort influences ocular motility in certain instances. It is important to note, however, that nonspecific factors such as arousal or effort may be separated from specific task effects. Results of all three experiments show that the verbalconceptual or visuospatial content of the question influences EMR independently of nonspecific factors that also may affect ocular motility. The longstanding conflict in the ocular motility literature between specific cognitive processing explanations (e.g., Lorens \& Darrow, 1962) and nonspecific-factor explanations (e.g., Amadeo \& Shagass, 1963) for differences in eye movement activity may be understood in light of the present findings that both kinds of factors may play simultaneous and independent roles in determining ocular motility.

Even though theoretical issues are far from being resolved, the present results provide a starting point for further research into the relationship between ocular motility and cognitive processes. In particular, the present study shows that the task-specific ocular motility effect, unlike the eye movement direction phenomenon, is a robust and stable effect that is amenable to systematic investigation.

\section{REFERENCES}

Amadeo, M., \& Gomez, E. Eye movements, attention and dreaming in subjects with lifelong blindness. Canadian Psychiatric Association Journal, 1966, 11, 501-507.

Amadeo, M., \& Shagass, C. Eye movements, attention and hypnosis. Journal of Nervous and Mental Disorders, 1963, 136, 139-145.

ATwoon, G. E. An experimental study of visual imagination and memory. Cognitive Psychology, 1971, 2, 290-299.

Bower, G. H. Analysis of a mnemonic device. American Scientist, $1970,58,496-510$.

Brady, J. P., \& Rosne R, B. S. Rapid eye movements in hypnotically induced dreams. Journal of Nervous and Mental Disorders, 1966, 143, 28-35.

Brooks, L. R. Spatial and verbal components of the act of recall. Canadian Journal of Psychology, 1968, 22, 349-368.

Brown, B. B. Visual recall ability and eye movements. Psychophysiology, 1968, 4, 300-306.

Crosby, E. C. Relations of brain centers to normal and abnormal eye movements in the horizontal plane. Journal of Comparative Neurology, 1953, 99, 437-479.

DAY, M. E. An eye movement phenomenon relating to attention, thought and anxiety. Perceptual and Motor Skills, 1964, 19, 443-446.

Ehrlichman, H., \& Weinberger, A. Lateral eye movements and hemispheric asymmetry: A critical review. Psychological Bulletin, 1978, 85, 1080-1101.

Ehrlichman, H., Weiner, S. L., \& Baker, A. H. Effects of verbal and spatial questions on initial gaze shifts. Neuropsychologia, 1974, 12, 265-277.

Heвs, D. O. Concerning imagery. Psychological Review, 1968, 75, 466-477.

Нısсоск, M. Effects of examiner's location and subject's anxiety on gaze laterality. Neuropsychologia, 1977, 15, 409-416.

KINSBOURNE, M. Eye and head turning indicates cerebral lateralization. Science, 1972, 176, 539-541.

Kinsbourne, M. Direction of gaze and distribution of cerebral thought processes. Neuropsychologia, 1974, 12, 279-281.

Kocel, K., Galin, D., Ornstein, R., \& Merrin, E. L. Lateral eye movement and cognitive mode. Psychonomic Science, 1972, 27, 223-224.

Lorens, S. A., \& Darrow, C. N. Eye movements, EEG, ESR, and EKG during mental multiplication. Electroencephalography and Clinical Neurology, 1962, 14, 739-746.

MARKS, D. F. Individual differences in the vividness of visual imagery and their effect on function. In P. W. Sheehan (Ed.), The function and nature of imagery. New York: Academic Press, 1972.

Moone, C. S. Control of the memory image. Psychological Review Monograph Supplement, 1903, 4(1, Whole No. 17), 277-306.

RAYNER, K. Eye movements in reading and information processing. Psychological Bulletin, 1978, 85, 618-660.

Robinson, D. A. Eye movement control in primates. Science, $1968,161,1219-1224$.

Singer, J. L., Greenberg, S., \& Antrobus, J. S. Looking with the mind's eye: Experimental studies of ocular motility during daydreaming and mental arithmetic. Transactions of the New York Academy of Sciences, 1971, 3, 694-709.

Teitelbaum, H. A. Spontaneous rhythmic ocular movements. Neurology, 1954, 4, 350-354.

TotTen, E. Eye-movement during visual imagery. Comparative Psychology Monograph, 1935, 11, No. 3 (Serial No. 53).

Weiner, S. L., \& EhrLichman, H. Ocular motility and cognitive process. Cognition, 1976, 4, 31-43.

Weitzenhoffer, A. M., \& Brockmeier, J. D. Attention and eye movements. Journal of Nervous and Mental Disorders, $1970,151,130-142$. 